\title{
IMAGE FUSION FOR TRAVEL TIME TOMOGRAPHY INVERSION
}

\author{
Linan Liu ${ }^{12}$ \\ Xiaomeng Zhao ${ }^{3}$ \\ Xinghui Yin ${ }^{1}$ \\ Muhammad Aqeel Ashraf ${ }^{45}$ \\ ${ }^{1}$ College of Computer and Information, Hohai University, Nanjing 210098, China \\ ${ }^{2}$ The Fourth Department, Bengbu Naval Petty Officer Academy, Bengbu 233012, China \\ ${ }^{3}$ College of Information Engineering, Anhui Science and Technology University, Chuzhou 233100, \\ China \\ ${ }^{4}$ Department of Geology, Faculty of Science, University Of Malaya 50603 Kuala Lumpur, Malaysia \\ ${ }^{5}$ Faculty of Science \& Natural Resources, Universiti Malaysia Sabah, 88400 Kota Kinabalu, Sabah, \\ Malaysia
}

\begin{abstract}
The travel time tomography technology had achieved wide application, the hinge of tomography was inversion algorithm, the ray path tracing technology had a great impact on the inversion results. In order to improve the SNR of inversion image, comprehensive utilization of inversion results with different ray tracing can be used. We presented an imaging fusion method based on improved Wilkinson iteration method. Firstly, the shortest path method and the linear travel time interpolation were used for forward calculation; then combined the improved Wilkinson iteration method with super relaxation precondition method to reduce the condition number of matrix and accelerate iterative speed, the precise integration method was used to solve the inverse matrix more precisely in tomography inversion process; finally, use wavelet transform for image fusion, obtain the final image. Therefore, the ill-conditioned linear equations were changed into iterative normal system through two times of treatment and using images with different forward algorithms for image fusion, it reduced the influence effect of measurement error on imaging. Simulation results showed that, this method can eliminate the artifacts in images effectively, it had extensive practical significance.
\end{abstract}

Keywords: tomography; inversion algorithm; wavelet transform; image fusion

\section{INTRODUCTION}

Cross well tomography technology is an effective method in geophysical prospecting, it uses the observed data acquired through the omnidirectional scanning of cross well medium, to get optimization inversion calculation results, ultimately get the image which can reflect cross well internal medium structure changes. It can solve many problems in engineering geology, it has been widely used in engineering geological exploration, such as the remaining oil distribution and reservoir precise description of oil and gas field, looking for inter well blind metallic ore or ore reserves prediction and so on. According to the projection data type, cross well tomography can be divided into the travel time tomography, waveform tomography and amplitude tomography etc. Among them, the travel time tomography method has several advantages of simple principle, less interference factors and the efficient calculation, so it occupies dominant position in cross well tomography application[1-7]. Although the cross well tomography technology has made some progress in theory study, model experiment, detection equipment, observation system, tomography algorithm, parameter inversion and geological interpretation, but at present it is still difficult to meet the practical application needs. This is mainly because in the calculation process of speed tomography, it has to solve these three problems: (1) calculation of ray tracing time in theory; (2) Calculation of the Jacobi matrix; (3) Solving sparse, ill conditioned equations, that is linearized inversion problem. There is a direct relationship between the first two problems and forward algorithms by ray tracing method, however, the geological conditions were varied, it had the lack of widely 
applied methods for tomography. Among them, the short path method proposed by Moser [8] and the linear travel time interpolation (LTI) by Asawaka and Kawanaka[9] are better. The short path method advantage is that, in any complex velocity medium condition, it can simulate minimum travel time (including first wave and diffracted wave) ray between each of the shot point / receive point, it does not need to smooth velocity model or use the virtual observation data. While the linear travel time interpolation method (LTI) has the advantages of high precision, fast speed, it is the most widely used ray tracing method in recent years. Therefore, in order to minimize artifacts, highlight the true anomaly area, we can use be different ray tracing methods on the same measuring objects to get the tomographic image, then use wavelet transform fusion method, it can improve the SNR of the tomographic images.

Therefore the first two problems have been solved, the third question cannot be ignored. According to the characteristics of the research object and method, ultimately the core of tomography is attributed to the solving of sparse, ill-conditioned, mixed set or incompatible linear equations. Therefore, it would have direct bearing on the success or failure of tomographic imaging, the accurate numerical solution and stability of inversion algorithm are necessary conditions of tomography realization. So far the effective inversion algorithms mainly include: $\mathrm{Cao}[10]$ proposed self-incentive simultaneous algebraic reconstruction algorithm(SASIRT) which was suitable for ray distribution was not uniform or measurement error was larger, however, it had slow calculation speed and low accuracy; Saad and Schultz[11] proposed generalized minimal residual method(GMRES) which could be used for solving non symmetric linear equations, the calculation process would not be interrupted generally until obtaining the exact solution, nevertheless, there may be not converge; Van and Vorst[12] proposed the double stable conjugate gradient method (BICGSTAB), which could be used for solving linear equations whose coefficient matrix was asymmetric, it used short recursive method to reduce residual progressively, so the advantage was it occupied less memory, but the convergence was irregular, the convergence rate may be amplified severely under the condition of finite precision; LSQR with damping factor method (DLSQR) proposed by Yang[13], it improved the inversion precision effectively, avoided numerical instability of LSQR algorithm when the measurement error was large, it was especially suitable for solving the equations whose coefficient matrix was large and sparse, compared with other iteration method, it could obtain faster convergence rate and better acceptable results in solving singular or illconditioned problems, currently it is practical inversion method which was most commonly used, however, the occupation of computer memory was large and accuracy should be further improved. In recent years, artificial intelligence method was also applied to the inversion algorithm[14], such as Simulated Annealing(SA) or Genetic Algorithm(GA), but it had strong dependence on the initial model, easily influenced by random disturbance, the distribution reconstruction effect on slightly more complex medium was poor.
According to this, comprehen sive utilization of inversion results with different ray tracing can be used[15-17]. Firstly, we use the shortest path method and the linear travel time interpolation method for forward calculation respectively, the ray travel time and Jacobi matrix are obtained; then presenting the improved Wilkinson iteration method to solve the sparse, ill conditioned equations, getting the inversion images with different forward methods; finally, the wavelet transform algorithm is used for image fusion[18]. The performance of the inversion algorithm and the fusion image resolution with new method are improved, it can meet the needs of practical application better.

\section{METHODOLOGY}

\section{THE IMPROVED WILKINSON ITERATION METHOD}

Because the distribution of transmitter and receiver is restricted by the detection of object space position, there are a lot of discrete grids which have no ray passed through, it results in projection data incomplete. So the coefficient matrix obtained from forward calculation is usually sparse, ill-conditioned, large scale or singular. Improved Wilkinson iteration method is good at solving ill-conditioned linear equations system in theory, it will control the iterative convergence error under a very small scale, meanwhile ensure fast convergence rate; iteration may be completed even within two paces at times. However, Wilkinson iteration method requires coefficient matrix is positive definite, which means the coefficient matrix obtained from forward calculation should be treated before using Wilkinson iteration method.

Consider the linear equation

$$
\mathrm{Ax}=\mathrm{b}
$$

In which, denotes the coefficient matrix, it is composed of ray length from each discrete grid; is an unknown vector, it represents the slowness vector from each grid cell; B represents a vector which composed of all ray travel time.

In order to get the positive definite coefficient matrix, both sides in the equation (1) are multiplied by, equation(1) is changed into equivalent equation (2).

$$
\mathrm{A}^{\mathrm{T}} \mathrm{Ax}=\mathrm{A}^{\mathrm{T}} \mathrm{b}
$$

In which, represents transposition of, is symmetric and positive definite, so it can meet the requirement of Wilkinson iteration. However, the condition number of is square of 's, when matrix A is ill-conditioned, small measurement error interference will bring greater calculation error, the convergence rate is very slow. Therefore, it is necessary to reduce the condition number of the coefficient matrix A.

Using the super relaxation preprocessing method, make, then the equation (1) changes into:

$$
\left(\mathrm{W}^{-\mathrm{T}} \mathrm{AW}^{-1}\right) \mathrm{Wx}=\mathrm{W}^{-\mathrm{T}} \mathrm{b}
$$


In which, is preprocessing factor matrix. As the structure of and is getting similar, the condition number of becomes small. So the specific selection method is as follows:

$$
\begin{gathered}
D_{i j}=\operatorname{diag}\left(\sqrt{\frac{A_{i j}}{\omega}}\right), \quad(i=j) \\
C=D^{-1}\left(D^{2}+L\right) \\
M=\frac{1}{2-\omega} C^{T} C
\end{gathered}
$$

Among them, is a diagonal matrix, is an upper triangular matrix, is strictly upper triangular matrix of , is relaxation factor, which ranges from $0<\omega<2$.

Make $\widetilde{A}=\mathrm{W}^{-\mathrm{T}} A \mathrm{~W}^{-1}, \tilde{\mathrm{x}}=\mathrm{Wx}, \tilde{\mathrm{b}}=\mathrm{W}^{-\mathrm{T}} \mathrm{b}$. Therefore the original equation(1) is changed into equivalent equation(7).

$$
\widetilde{\mathrm{A}} \tilde{\mathrm{x}}=\tilde{\mathrm{b}}
$$

The condition number of coefficient matrix in equation (7) is much smaller than the original matrix A's, then both sides in the equation (7) are multiplied by $\widetilde{\mathrm{A}}^{\mathrm{T}}$, so equation(7) is changed into:

$$
\widetilde{\mathrm{A}}^{\mathrm{T}} \widetilde{\mathrm{A}} \tilde{\mathrm{x}}=\widetilde{\mathrm{A}}^{\mathrm{T}} \tilde{\mathrm{b}}
$$

Right now the matrix $\widetilde{\mathrm{A}}^{\mathrm{T}} \widetilde{\mathrm{A}}$ in equation (8) becomes symmetric and positive definite, and the condition number of coefficient matrix is smaller than equation (2).

So the solution of equation (1) is converted to solving equation (8) and getting the slowness vector $\mathrm{x}$ from solving $\tilde{\mathrm{x}}=\mathrm{Wx}$. The solving of $\tilde{\mathrm{x}}=\mathrm{Wx}$ is very simple, so next we mainly discuss solution of equation (8). In order to solving it more precisely and faster, a small parameter can be introduced to form an improved Wilkinson iteration method.

$$
\left\{\begin{array}{c}
\mathrm{z}_{\mathrm{k}}=\left(\widetilde{\mathrm{A}}^{\mathrm{T}} \widetilde{\mathrm{A}}+\sigma^{2} \mathrm{I}\right)^{-1}\left(\widetilde{\mathrm{A}}^{\mathrm{T}} \mathrm{b}-\widetilde{\mathrm{A}}^{\mathrm{T}} \widetilde{\mathrm{A}} \tilde{\mathrm{x}}_{\mathrm{k}}\right) \\
\tilde{\mathrm{x}}_{\mathrm{k}+1}=\tilde{\mathrm{x}}_{\mathrm{k}}+\mathrm{z}_{\mathrm{k}}
\end{array}\right.
$$

In which, $\tilde{\mathrm{x}}_{0}=0, \sigma$ is a small arbitrary constant, $\mathrm{I}$ is a unit matrix.

With the treatment of super relaxation precondition and Wilkinson iteration method, the ill conditioned linear equations is changed into normal system, then use equation (9) to get iterative solution.

\section{USING THE PRECISE INTEGRATION METHOD TO GET INVERSE MATRIX}

Although the matrix is improved, but the coefficient matrix A may be singular or singular values are very small, it may cause we cannot get the precise solution by calculating inverse matrix $\widetilde{A}^{\mathrm{T}} \widetilde{\mathrm{A}}+\sigma^{2}$ I directly. Therefore, the precise integration thought is used to get inverse matrix which is more precise.

Make $F=\widetilde{A}^{T} \widetilde{A}+\sigma^{2} I, H(t)=\int_{0}^{t} \exp (-F s) d s$, then
$H(t)=\int_{0}^{t} \exp (-F s) d s=-\left.F^{-1} \exp (-F s)\right|_{0} ^{t}=-F^{-1} \exp (-F t)+F^{-1}$

Because $\mathrm{F}$ is positive definite, when $\mathrm{t} \rightarrow+\infty$, $\exp (-\mathrm{Ft}) \rightarrow 0$ so $\mathrm{F}^{-1}=\mathrm{H}(+\infty)$. Make $\mathrm{B}=-\mathrm{F}$, then

$$
\begin{gathered}
H(2 t)=\int_{0}^{2 t} \exp (B s) d s=\int_{0}^{t} \exp (B s) d s+\int_{t}^{2 t} \exp (B s) d s= \\
=(I+\exp (B t)) \int_{0}^{t} \exp (B s) d s
\end{gathered}
$$

That is

$$
H(2 t)=(I+\exp (B t)) H(t)
$$

Equation (10) provides the recursive algorithm for matrix $\mathrm{F}^{-1}$, then use the precise integration thought, take a small step $\Delta \mathrm{t}$, make

$$
H_{j}=H\left(2^{j} \Delta t\right) \quad(j=0,1, \ldots)
$$

Then

$$
\left\{\begin{array}{c}
\mathrm{H}_{0}=\Delta \mathrm{t}\left(\mathrm{I}+\sum_{\mathrm{i}=1}^{4} \frac{(\mathrm{B} \Delta \mathrm{t})^{\mathrm{i}}}{\mathrm{i} !}\right) \\
\mathrm{H}\left(2^{\mathrm{j}} \Delta \mathrm{t}\right)=\prod_{\mathrm{i}=0}^{\mathrm{j}-1}\left(\mathrm{I}+\exp \left(\mathrm{B} \cdot 2^{\mathrm{i}} \cdot \Delta \mathrm{t}\right)\right) \mathrm{H}_{0} \quad(\mathrm{j}=1,2, \ldots)
\end{array}\right.
$$

Calculating $\exp \left(\mathrm{B} \cdot 2^{\mathrm{i}} \cdot \Delta \mathrm{t}\right)$ with the precise integration method, first do Taylor expansion for $\exp (B \Delta t)$, because $\Delta t$ is very small, it can only retain the first several series in Taylor expansion.

$$
\exp (B \Delta t) \approx I+\sum_{i=1}^{4} \frac{(B \Delta t)^{i}}{i !}
$$

Make

$$
\left\{\begin{array}{c}
\mathrm{T}^{(0)}=\sum_{\mathrm{i}=1}^{4} \frac{(\mathrm{B} \Delta \mathrm{t})^{\mathrm{i}}}{\mathrm{i} !} \\
\mathrm{T}^{(\mathrm{i}+1)}=2 \mathrm{~T}^{(\mathrm{i})}+\left(\mathrm{T}^{(\mathrm{i})}\right)^{2}
\end{array}\right.
$$

The benefit is that, it can separate large variables from small in the calculation, avoid the small variables are ignored when they are added with large, ensure the calculation accuracy.

So

$$
\exp \left(\mathrm{B} \cdot 2^{\mathrm{i}} \cdot \Delta \mathrm{t}\right)=I+\mathrm{T}^{(\mathrm{i})} \quad(\mathrm{i}=1,2, \ldots)
$$

From equation (10) and (15), we can get

$$
\mathrm{H}_{\mathrm{j}+1}=\left(\mathrm{I}+\exp \left(\mathrm{B} \cdot 2^{\mathrm{i}} \cdot \Delta \mathrm{t}\right)\right) H_{0} \quad(j=1,2, \ldots)
$$

When $\mathrm{j}$ is large enough, we can get more accurate inverse matrix $\mathrm{F}^{-1}$. Meanwhile, the iterative process is equivalent to multiplying the integral region of $\mathrm{H}(\mathrm{t})$, the convergence is carried on with steps of exponential $2^{\mathrm{k}}$, hence, the calculation efficiency is very high.

\section{WAVELET TRANSFORM}

Wavelet transform is a time-frequency localization 
analysis, it has higher frequency resolution and low time resolution in low frequency part of signal, has low frequency resolution and higher time resolution in higher frequency part of signal. Therefore, the process of image fusion based on wavelet transform is: firstly, the preparative fusion image are decomposed into low frequency and high frequency coefficients, then the high or low frequency coefficients of image are fused to form wavelet Pyramid new structure according to some fusion rules, finally, the fusion image is obtained by wavelet reconstruction. The concrete steps of wavelet coefficients fusion is as follows.

(1) Calculation of high frequency coefficients edge active measure:

Regarding image A, the high frequency coefficients edge active measure:

$$
\mathrm{E}_{\mathrm{j}}^{\varepsilon}(\mathrm{A}, \mathrm{p})=\left[\sum_{\mathrm{i}=1}^{\mathrm{n}} \mathrm{W}_{\mathrm{i}} * \mathrm{~d}_{\mathrm{j}}^{\varepsilon}(\mathrm{A}, \mathrm{p})\right]^{2}
$$

Low frequency coefficients edge active measure:

$$
\mathrm{E}(\mathrm{A}, \mathrm{p})=\left[\sum_{\mathrm{i}=1}^{\mathrm{n}} \mathrm{W}_{\mathrm{i}} * \mathrm{C}_{\mathrm{L}}(\mathrm{A}, \mathrm{p})\right]^{2}
$$

Where, $\mathrm{W}_{\mathrm{i}}$ is Frei-chen template, ${ }^{\star}$ represents convolution computation, $C_{L}(A, p)$ and $d_{j}^{\varepsilon}(A, p)$ represent low frequency coefficient and high frequency coefficient respectively, $A$ represents decomposition, $\mathrm{p}$ represents the pixel position. Similarly we can get the edge active measure coefficient for image B.

\section{(2) Synthesis coefficient}

The high or low frequency coefficients of image F fused by images A and B are as follows.

High frequency coefficient:

$$
d_{j}^{\varepsilon}(F, p)=d_{j}^{\varepsilon}(A, p) M_{j}^{\varepsilon}(A, p)+d_{j}^{\varepsilon}(B, p) M_{j}^{\varepsilon}(B, p)_{(19)}
$$

Among them, if $E_{j}^{\varepsilon}(A, p) \geq E_{j}^{\varepsilon}(B, p), M_{j}^{\varepsilon}(A, p)=1$; otherwise

$$
\begin{gathered}
M_{j}^{\varepsilon}(A, p)=0, M_{j}^{\varepsilon}(B, p)=1- \\
-M_{j}^{\varepsilon}(A, p), j=1,2,, \cdots, L
\end{gathered}
$$

Low frequency coefficient:

$$
C_{L}(F, p)=C_{L}(A, p) M_{L}(A, p)+C_{L}(B, p) M_{L}(B, p)
$$

Where, if $E(A, p) \geq E(B, p), M_{L}(A, p)=1$; otherwise

$$
\begin{gathered}
M_{L}(A, p)=0 \\
M_{L}(B, p)=1-M_{L}(A, p) .
\end{gathered}
$$

\section{THE STEPS OF IMPROVED ALGORITHM AND ANALYSIS OF CONVERGENCE}

\section{ALGORITHM STEPS BASED ON IMAGE FUSION}

Through above analysis, the steps using two kinds of ray tracing method to forward modeling, improved Wilkinson iteration inversion algorithm and wavelet transform fusion are as follows.

First step: The short path method and the linear travel time interpolation method are used to calculate the travel time and the Jacobi matrix;

Second step: Make pretreatment to equation (1) with the super relaxation iteration method, use equations (3) (4) (5) (6) to get (7), then both sides in the equation (7) multiply by $\widetilde{\mathrm{A}}^{\mathrm{T}}$ to get equation (8), then the coefficient matrix is positive definite and condition number is smaller;

Third step: Calculate inverse matrix of $\widetilde{\mathrm{A}}^{\mathrm{T}} \widetilde{\mathrm{A}}+\sigma^{2}$ using the precise integration method, obtain more accurate inverse matrix by iteration using the equation (16);

Fourth step: Select small parameter $\sigma$ and step $\Delta \mathrm{t}$, use improved Wilkinson iteration equation (9) to obtain $\tilde{\mathrm{x}}$, then get slowness vector $\mathrm{x}$ by solving $\tilde{\mathrm{x}}=\mathrm{Wx}$, accomplish inversion calculation.

Last step: Fusion with using wavelet transform, the fusion image is obtained with high SNR.

\section{ANALYSIS OF ALGORITHM CONVERGENCE}

Reorganize the iteration formula (9), $\tilde{\mathrm{x}}_{\mathrm{k}+1}=\mathrm{G} \tilde{\mathrm{x}}_{\mathrm{k}}+\mathrm{g}$ is obtained, in which, $\mathrm{G}=\mathrm{I}-\left(\widetilde{\mathrm{A}}^{\mathrm{T}} \widetilde{\mathrm{A}}+\mathrm{qI}\right)^{-1} \widetilde{\mathrm{A}}^{\mathrm{T}} \widetilde{\mathrm{A}}$,

$g=\left(\widetilde{A}^{\mathrm{T}} \widetilde{\mathrm{A}}+\mathrm{qI}\right)^{-1} \widetilde{\mathrm{A}}^{\mathrm{T}} \tilde{\mathrm{b}}$. Because the equivalent coefficient matrix $\widetilde{\mathrm{A}}^{\mathrm{T}} \widetilde{\mathrm{A}}$ after deformation of original equation is positive definite, make its characteristic values $\alpha_{i}(i=1,2, \ldots)$, then characteristic values of $G$ are $1-\frac{\alpha_{i}}{\alpha_{i}+\sigma^{2}}>0$. So the spectral radius of $G$ is

$$
\rho(G)=\max \left(1-\frac{\alpha_{i}}{\alpha_{i}+\sigma^{2}}\right)=\max \left(\frac{\sigma^{2}}{\alpha_{i}+\sigma^{2}}\right)<1
$$

Therefore, from equation $(21)$ it can be seen, $\rho(G)<1$ with arbitrary variable $\sigma$, the iteration formula (9) is always convergent. So we can obtain the iteration result in an acceptable range all along.

\section{ANALYSIS OF MODEL SIMULATION}

For analyzing the effect of new inversion algorithm in various media conditions better, we design three models: independent anomaly model, layered area model and abnormal S shape model. Use PIW algorithm in this paper for inversion, wavelet transform algorithm for image fusion, compare and analyze the inversion results from 
imaging precision, time efficiency and the sensitivity of measurement error.

\section{THE TEST OF THEORETICAL MODEL}

The velocity distribution model of layered medium is showed in Figure 1. Assume a model which is abnormal velocity $S$ shape in homogeneous background, the velocities in abnormal zone and homogeneous background are $600 \mathrm{~m} / \mathrm{s}, 1000 \mathrm{~m} / \mathrm{s}$ respectively. The size of model is $\mathrm{m}$, the source points number is 85 , first source point is located at a depth of $0.2 \mathrm{~m}$, the interval of source points is $0.1 \mathrm{~m}, 85$ source points are distributed evenly in the hole. The position coordinate of right hole surface is $(10,0)$, put the receiver points in the right hole. Similarly, the number is 85 , first receiver point is located at a depth of $0.2 \mathrm{~m}$, the interval of receiver points is $0.1 \mathrm{~m}, 85$ receiver points are distributed evenly in the hole also.

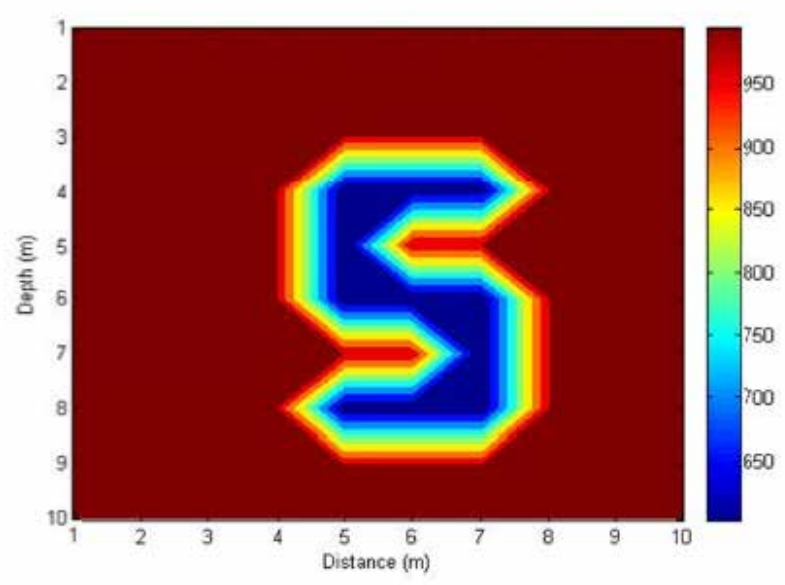

Figure 1. Velocity distribution of Model 1

The shortest path method and the linear travel time interpolation are used for forward ray tracing, add the forward ray travel time $15 \%$ random error, then use improved Wilkinson iteration algorithm for inversion, the value of is chosen as 0.005, as shown in Figure 2.

(a)

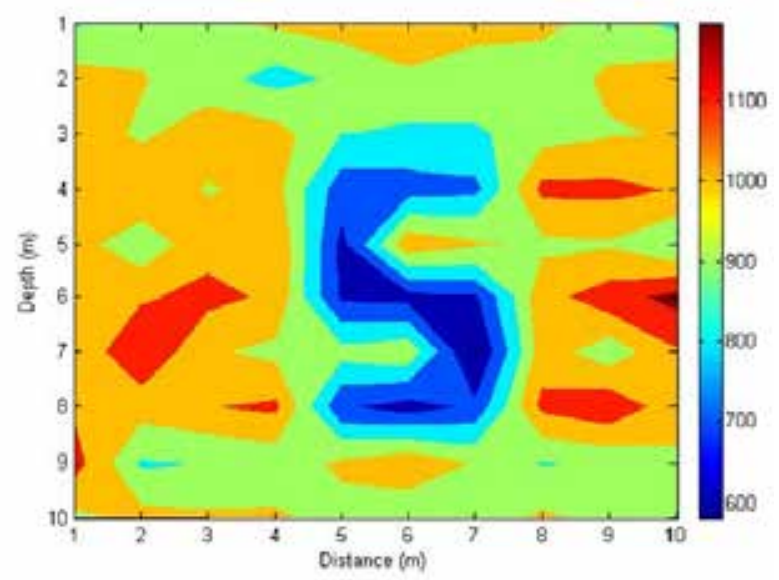

(b)

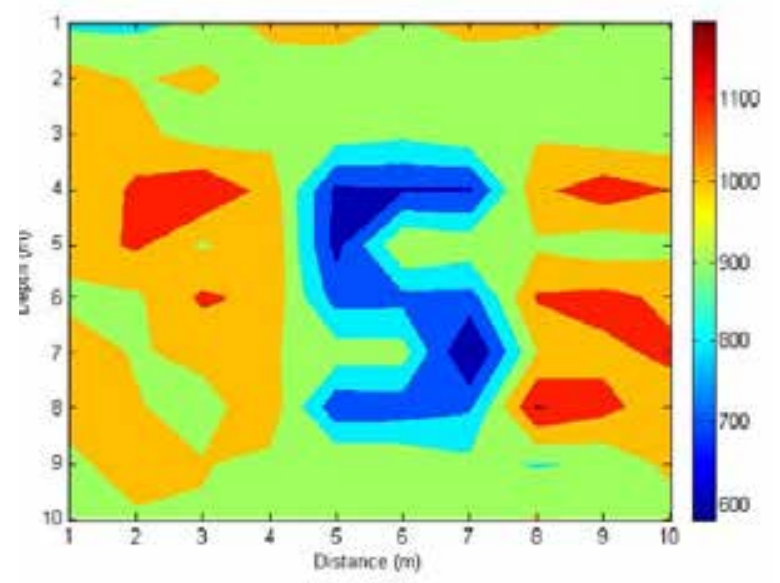

(c)

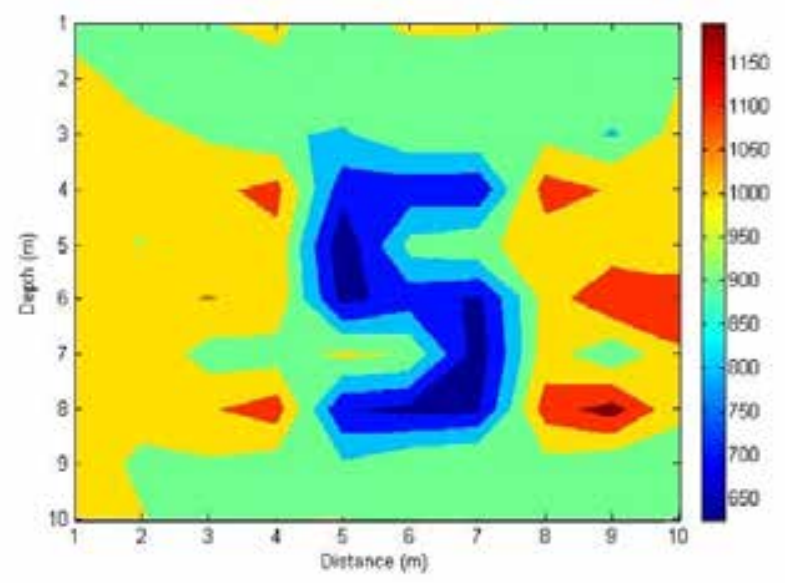

Figure 2. Velocity distribution inversion of Model 1. (a) Shortest path method (b) LTI method (c) Fused image

After adding $15 \%$ random error, the low velocity zone in Model can be reflected basically clearly based on two kinds of ray tracking methods, it illustrates two methods are effective for such cross well medium distribution such as karst cave. But there are some high-speed regional artifacts in uniform region, although we can easily distinguish the theoretical model, but it will have adverse effects for the actual geological survey. After fusion low speed region is high-contrast, and it eliminates some high speed artifacts, so it can satisfy the demand of imaging on abnormal regional location and size identification better.

Considering there are more layered velocity model occurred in superficial stratum practical application, a hierarchical model is proposed, high and low velocity anomaly area are shown in Figure 3.

We still use shortest path method and the linear travel time interpolation for forward ray tracing, add the forward ray travel time $15 \%$ random error, then use improved Wilkinson iteration algorithm for inversion, the value of $\sigma$ is chosen as 0.005, as shown in Figure 4. 


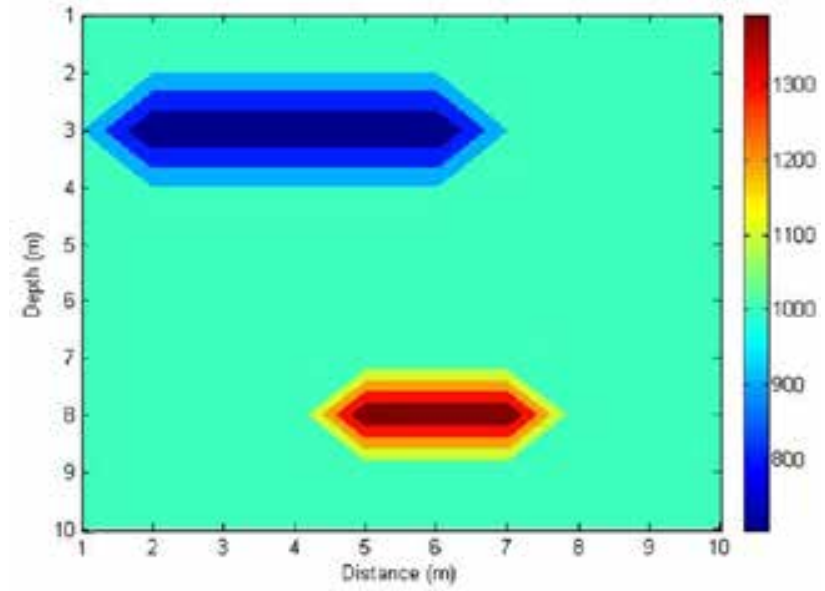

Figure 3. Layered medium velocity distribution of Model 2

(a)

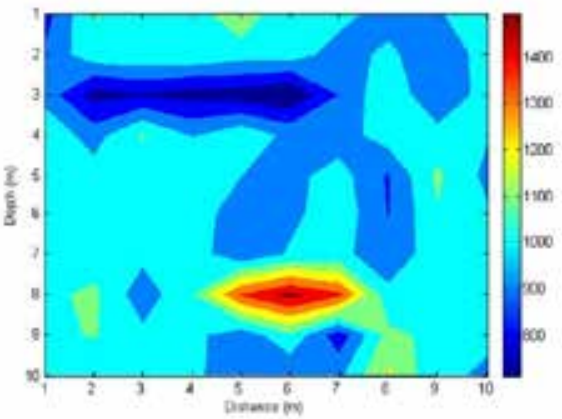

(b)

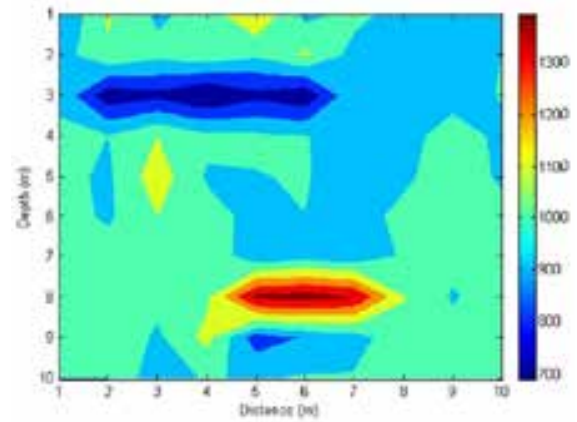

(c)

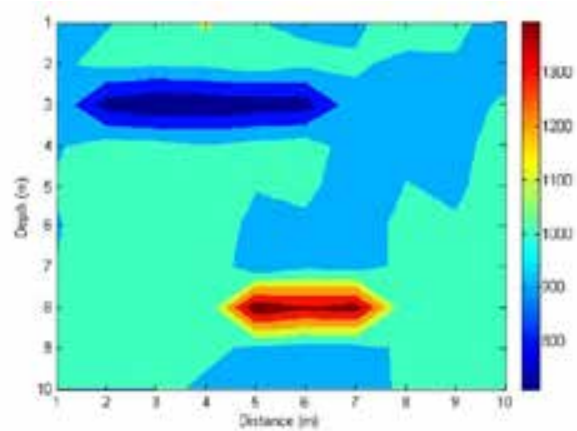

Figure 4. Velocity distribution inversion of Model 2. (a) Shortest path method (b) LTI method (c) Fused image
After adding 15\% random error, the layered distribution from top to bottom between wells in two images still can be reflected, it illustrates the effectiveness of two methods. However, artifacts appeared in background of homogeneous regions affect resolution of true anomaly area. After fusion low speed region is high-contrast, edge distribution is clear, it can depict layered distribution of different speed basic faithfully. Because there are many layered distribution in the actual geological detection, imaging for layered media plays a very important role in the practical application, this reflect the practicability of the proposed method.

Using the abnormal regional model and programming ideas in reference [19], modifying the program according to improved Wilkinson iteration algorithm. Regional location, launch or receiving point coordinates are given in Figure 5. Using shortest path method and the linear travel time interpolation for forward ray tracing, inversion image is got after fusion. To reflect inversion image clearly, the original model and inversion image are put together in a chart. (a)

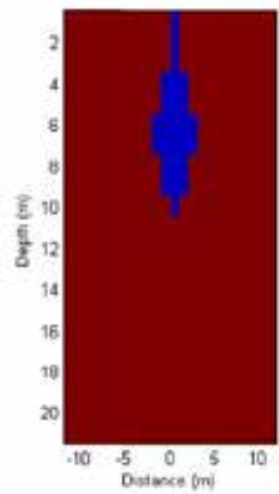

(c) (b)

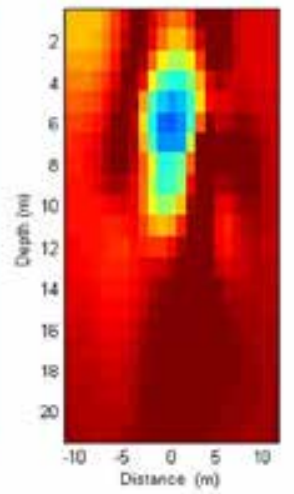

(d)
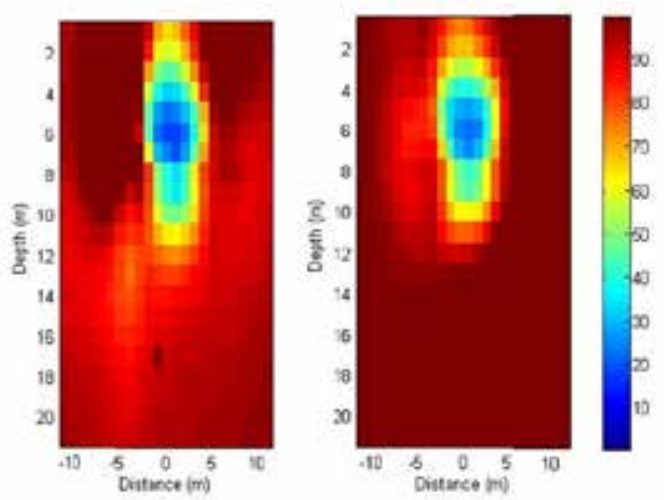

Figure 5. Velocity distribution inversion. (a) velocity distribution of Model 3 (b) Shortest path method (c) LTI method (d) Fused image

It can be seen from Figure 5, after fusing the images of shortest path method and the linear travel time interpolation, although some areas is abnormal, which is determined by the error introduced into the calculation of false information, however the whole image can still ensure the resolution effect very well. It can improve the accuracy and reliability of inversion image with using different methods which provide redundant 
information, and the fused image contains more comprehensive, abundant information.

\section{THE INVERSION OF REAL DATA}

In order to verify the effect of fusion imaging method, using the following practical cross-well seismic data to tomography[20]. The area of seismic data acquisition locate near a power plant in Paineiras Florida USA , in 2002 Berkeley National Laboratory collected seismic data by using the multi well observation, the purpose of the study is to analyze the pollution liquid distribution in the near surface. AjoFranklin used adaptive grid-travel time tomography method to get velocity inversion. We still use shortest path method and the linear travel time interpolation for forward ray tracing, then use improved Wilkinson iteration algorithm for inversion, as shown in Figure 6. As can be seen from the graph, the fusion image can depict of the two low velocity anomaly zone in deep stratum more accurately, this part may be caused by polluting liquid diffusion in deep strata lateral, in comparison to the original image[20], the two low velocity anomaly areas in fused image are depicted more clearly.

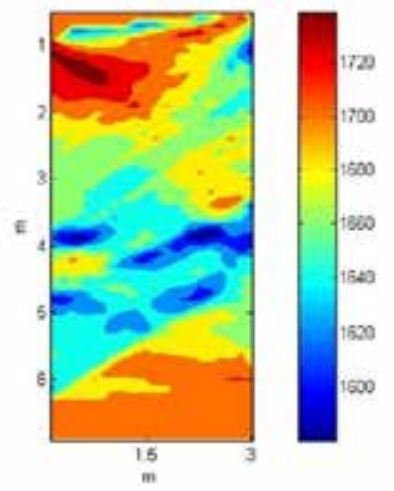

Figure 6. The inversion image of real data

\section{CONCLUSION}

There are many ray tracing methods used in the velocity tomography imaging, different methods show their merits under different geological conditions. Therefore, the using two kinds of commonly used ray tracing methods are used for forward calculation to obtain travel time and the Jacobi matrix; the inversion algorithm is we combined the improved Wilkinson iteration method with super relaxation precondition method to get the iterative slowness values in cross well, the precise integration method was used to solve inverse matrix more precisely in Wilkinson iteration process, finally, wavelet transform is used for image fusion, it effectively eliminates the artifacts in the image, improves the velocity anomaly area recognition in tomography image.

\section{REFERENCES}

1. H.M. Benz, B.A. Chouet, P.B. Dawson, J.C. Lahr, R.A. Page, and J.A. Hole, "Three dimensional P and S wave velocity structure of Redoubt Volcano”. Journal of Geophysical Research,vol.101,pp. 8111-8128, 1996.

2. L. Huang, Z.Y. Huang and Y. Wang, "The inversion algorithm of ultrasonic tomography with concrete structure”. Journal of Hunan University (Natural sciences), vol. 33, no. 5, pp. 26- 30, 2006.

3. T. Nemeth, E. Normark, and F.H. Qing, "Dynamic smoothing in cross well travel time tomography". Geop hysics,vol.62,no.1,pp.168-176,1997.

4. H. Maurer, A.G. Green, and K. Holliger, "FullWaveform inversion of cross-hole radar data based on 2-D finite-difference time domain solutions of Maxwell's equations". IEEE transactions on geoscience and remote sensing,vol.72,no.5,pp.53-64, 2007.

5. S.C. Su, S.D. Wang and L. Wu, "Smoothing SIRT algorithm with cross well tomography". Journal of China University of Petroleum (Natural sciences), vol.25,no.6,pp.29-31, 2001.

6. C. C. Paige and M. A. Saunders, "Sparse linear equations and least squares problems". ACM Transactions on Mathematical Software, vol. 8,no.2,pp.195-209,1982.

7. N. Linde, A. Tryggvason, J.E. Peterson, and S.S. Hubbard, "Joint inversion of cross hole radar and seismic travel times acquired at the South Oyster Bacterial Transport Site”. Geophysics, vol. 73, no.4,pp.39-50, 2008.

8. Moser T J, "Shortest path calculation of seismic rays". Geophysics, vol. 56,no.1,pp. 59 67,1991.

9. Asakawa, Eiichi and Kawanaka, Taku, "Seismic ray tracing using linear travel time interpolation”. Geophysical Prospecting,vol.41,no.l,pp.99 112,1993.

10. J.X. Cao and Z.Q. Yan. "Estimation of seismic crosshole travel time tomography resolution". Journal of Chengdu University of Science \& Engineering, vol.22,no.4,pp.95-101, 1995.

11. Y. Saad and M.H. Schultz. "GMRES: Generalized minimal residual algorithm for solving non-symmetric line system". SLAM J.SCI.STAT.COMPUT, vol.7, no.3,pp.856-869,1986.

12. G.L.G. Sleijpen and D.R. Fokkema, "Bicgstab (L) for linear equations involving unsymmetric matrices with complex spectrum". Electronic trasactions on numerical analysis, vol.1, pp.11 -32,1993. 
13. W.C. Yang and J.Y. Du, "A new algorithm for tomographic imaging and its application in engineering detection". Chinese Journal of Geophysics, vol.37,no.2,pp.239-244, 1994.

14. F. Boschetti, M.D. Dentith and R.D. List, "A fractalbased algorithm for detecting first arrivals on seismic traces”. Geophysics, vol.61,no.4,pp.1095-1102,1996.

15. C.X. Chen and H.D. Yin, "Using super relaxation preconditioned conjugate gradient method for solving large sparse equations". Science technology and Engineering, vol.10,no.10,pp. 2389-2394, 2010.

16. X.Y. WU, R. SHAO and Y.R. ZHU, "Iterative improvement of a solution for an ill conditioned system of linear equations based on a linear dynamic system". Computers and Mathematics with Applications,vol.44,pp.1109-1116,2002.

17. W.Z. Zhang and P.Y. Huang, "Precise iterative method for solving ill conditioned algebraic system". Applied Mathematics and Mechanics, vol.34,no.7,pp.736-741,2013.

18. Li H, Manjunath BS and Mitra SK, "Multisensor image fusion using the wavelet transform". Graphical Models and Image Processing,vol.57,no.3,pp.235 245,1995.

19. Adam Pidlisecky, Eldad Haber, and Rosemary Knight, "RESINVM3D: A 3D resistivity inversion package". Geophysics, vol.72,no.2, pp.H1-H10,2007.

20. Ajo-Franklin J B. "Using high resolution borehole geophysics for DNAPL detection and environmental site characterization", California:Department of Geophysics, Stanford University,2005

\section{CONTACT WITH THE AUTHORS}

Xinghui Yin

College of Computer and Information Hohai University Nanjing 210098

\section{CHINA}

Linan Liu

The Fourth Department Bengbu Naval Petty Officer Academy, Bengbu 233012

CHINA

$$
\text { Xiaomeng Zhao }
$$

College of Information Engineering Anhui Science and Technology University Chuzhou 233100

\section{CHINA}

Muhammad Aqeel Ashraf

Department of Geology, Faculty of Science University Of Malaya 50603 Kuala Lumpur MALAYSIA 